\title{
SITUACIÓN DE LA PRODUCCIÓN DE FRIJOL Y MAÍZ EN CONCEPCIÓN DE PILAS Y VERACRUZ DE PEJIBAYE, COSTA RICA ${ }^{1}$
}

\author{
Pablo Alvarez ${ }^{2}$, Hernando Cárdenas ${ }^{2}$
}

\begin{abstract}
RESUMEN
Situación de la producción de frijol y maíz en Concepción de Pilas y Veracruz de Pejibaye, Costa Rica. El estudio socioeconómico se llevó a cabo en la zona sur de Costa Rica, en el distrito de Pejibaye del cantón de Pérez Zeledón, mediante una encuesta a 80 productores escogidos aleatoriamente; en el mes de mayo de 1999. El 84\% de los agricultores poseen tierra propia para la producción, con unidades productivas en promedio de 14,5 ha. La mayoría de los productores de granos básicos diversifican con otras actividades agropecuarias, como tiquisque, café y pastos. El nivel de diversificación se incrementó con el tamaño de las unidades productivas. La mayor parte lo diversifica con la combinación maíz y frijol. El $90 \%$ de los productores que siembran maíz, tienen este cultivo durante la época veranera (diciembre a febrero), con un área promedio de 2,5 ha y con un rendimiento promedio de 1,9 t/ha. En el caso del frijol la siembra principal se da en la época inverniz (mayo a agosto), con un área promedio de 3 ha, y un rendimiento de $0,6 \mathrm{t} / \mathrm{ha}$. Para ambos granos, la tendencia es hacia la reducción de áreas y rendimientos. Tanto para maíz como para frijol, la mayor parte de la cosecha se destina a la venta, y en general se puede señalar que la producción de ambos granos resulta rentable en términos de la relación beneficio/costo, siendo más rentable la actividad frijolera. En los últimos años las inestabilidades climáticas han afectado los rendimientos de los granos básicos, lo cual ha llevado a los productores a incursionar en otras actividades productivas. Los productores han empezado a utilizar una serie de estrategias para contrarrestar estos efectos, tales como el uso de coberturas, y diversas prácticas culturales y agronómicas.
\end{abstract}

\begin{abstract}
Situation of corn and bean production at Pejibaye of Perez Zeledon, and Pilas of Buenos Aires, Costa Rica. The socioeconomic study was conducted at the southern zone of Costa Rica, in the district of Pejibaye, located in Perez Zeledón, through an inquiry of 80 producers aleatorily chosen. The information was obtained on May, 1999. About $84 \%$ are landowners, with productive units of an average of 14.5 ha. Most of the basic grains producers diversify with others farming activities, such as spoonflower, coffee and pasture. It was determined that the level of diversification increases with the size of the productive units. Also, within the subsystem of basic grains, most of them diversify it in combination with corn and bean. Ninety percent of the producers plant corn during summer time (December to February), with an average area of 2.5 ha and with an average production of $1.9 \mathrm{t} / \mathrm{ha}$. Regarding bean, the main crop comes on wintertime (May to August) with an average area of $3 \mathrm{ha}$, and a yield of de $0.6 \mathrm{t} / \mathrm{ha}$. For both grains, the tendency goes to the reduction of areas and product. For corn, as well as for the bean, most of the crop goes for market, and in general, that is to say that the production of both grains have an income-producing result in terms of cost/benefit, being more profitable the bean activity. During the latter years, the climatic instabilities have affected the output of basic grains, which has made the producers to turn into other productive activities. The producers have began to use some strategies in order to encounter these effects, such as the use of coverings, and some other cultural and agronomic practices.
\end{abstract}

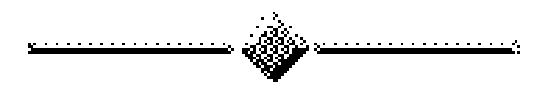

\section{INTRODUCCIÓN}

Este trabajo presenta los resultados de una investigación socioeconómica sobre el comportamiento de pe- queños y medianos productores de granos básicos en la región Brunca de Costa Rica. Dentro de esta región se seleccionó la zona de Pejibaye por su importancia en la producción de granos y por la presencia de pequeños y

1 Proyecto de investigación del Instituto para el Desarrollo y la Acción Social (IDEAS).

2 Instituto para el Desarrollo y la Acción Social (IDEAS)Tel: (506)234-9841 / 280-9635 Fax: (506)2349841. Apdo Postal 1465-2050 Email: aideas@racsa.co.cr. San José, Costa Rica. 
medianos productores dedicados a esta actividad desde hace varias décadas.

La tendencia del desarrollo económico de la región se orienta hacia la integración de tres actividades principales. En primer lugar la actividad agropecuaria, en segundo lugar la agroindustrial asociada a los principales productos agrícolas como son el café, palma aceitera, caña y piña. El tercer lugar es ocupado por la actividad turística, que aunque es la menos desarrollada, presenta un gran potencial en la generación de ingresos (Gamboa y Quiros, 1998). Sin embargo es notable la producción de granos básicos de la región, es muy importante en el abastecimiento del mercado nacional, donde siguen siendo esenciales en la dieta de los costarricenses (Murillo y Mora, 1996).

El distrito de Pejibaye se localiza en la zona sur de Costa Rica, pertenece al cantón de Pérez Zeledón y su extensión es de 205,9 km².

Existe una franja granera de maíz y frijol en Pejibaye, que abarca las comunidades de Zapote, San Martín, Guadalupe, San Fe, Veracruz, Las Delicias, y que se comunica al sur-este con las localidades de Guagaral, Concepción, Colinas y China del cantón de Buenos Aires (Alvarez y Mora, 1994).

La población de Pejibaye, sobre todo desde los años sesenta, está conformada principalmente por pequeños productores de café, maíz y frijol. Según el Censo Agropecuario de 1984, había una extensión de 1644,8 hectáreas ocupadas por 985 explotaciones. Un $18,9 \%$ se dedicaba a tierras de labranza, un $6,1 \%$ a cultivos permanentes, un $61,4 \%$ a pastos, y un $13,8 \%$ tierra no cultivada.

En la actualidad las principales actividades agropecuarias de la zona son el maíz, el frijol, el café y la ganadería.

\section{MATERIALES Y MÉTODOS}

El estudio se llevó a cabo en la región Brunca de Costa Rica, en el distrito de Pejibaye, representativo de la misma. Se definió una muestra de 80 productores escogidos aleatoriamente y distribuidos en 14 comunidades, a quienes se les distribuyó una encuesta de tipo socioproductivo. Para complementar la información recolectada se hicieron entrevistas estructuradas, dirigidas a productores de reconocida experiencia en la zona. Las encuestas las realizaron funcionarios del Ministerio de Agricultura y Ganadería (MAG) en la zona en el mes de mayo de 1999. El seguimiento y supervisión del proceso de campo y estadístico, así como la elaboración del presente artículo estuvo a cargo del Instituto para el Desarrollo y la Acción Social (IDEAS).

\section{RESULTADOS}

\section{Características socioeconómicas de los productores}

Características sociales del productor y su familia: el tamaño del grupo familiar es en promedio de cinco personas, hallándose familias con sólo una persona o núcleos de 12 miembros. El 50\% de las familias son jóvenes, en tanto cuentan con dos miembros adultos y tres menores de 12 años. Asociado a esto, la edad promedio del productor jefe de hogar es de 43 años.

Se ve claramente que la generación de ingresos fuera del ámbito de la finca (como peón o jornalero) como actividad principal, es muy reducida. Así, la mayoría de los varones dependen de los ingresos obtenidos de la actividad agropecuaria. Por otro lado, un $43 \%$ de las mujeres se dedica a actividades domésticas (Figura 1).

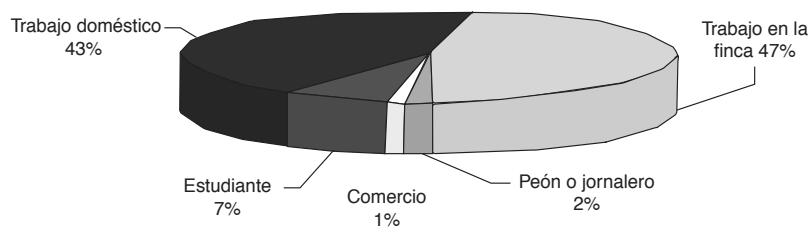

Fuente: IDEAS, datos de la investigación

Figura 1. Distribución de la población mayor de 12 años según actividad principal.

Tenencia de la tierra: La mayoría de productores (84\%) son propietarios de los terrenos, y dentro de éstos hay quienes trabajan bajo otras modalidades: alquilado, prestado, al medio o al tercio (este sistema consiste en que el dueño de la tierra recibe la mitad o la tercera parte de lo que se produce). Dentro de los productores sin tierra (16\%) están los adjudicatarios del Instituto de Desarrollo Agrario (IDA), y productores que deben recurrir a terrenos de terceros para producir, generalmente bajo el sistema de al medio, al tercio. En promedio las unidades productivas son de 14,5 ha.

Al establecer rangos de áreas, la distribución de productores muestra la tendencia que al aumentar el área de producción, hay un mayor porcentaje de productores que se sitúan dentro de los rangos superiores de tamaño de finca; a excepción de las fincas 
mayores de 50 hectáreas, las cuales poseen el menor porcentaje de productores (Cuadro 1).

Cuadro 1. Distribución porcentual de productores según tamaño de fincas.

\begin{tabular}{lc}
\hline Hectáreas & \% de productores \\
\hline 0 a 5 & 24 \\
5 a 10 & 29 \\
10 a 50 & 35 \\
Más de 50 & 12 \\
\hline
\end{tabular}

\section{Características agroecológicas}

En ambas comunidades los suelos pertenecen al orden de los ultisoles (Tropohumults), con pendiente de 30 a $60 \%$, cuyo uso corresponde a cultivos anuales (granos básicos, tubérculos), y en menor medida a perennes y ganadería. El clima de la zona se clasifica como tropical lluvioso. Se encuentran zonas climáticas que incluyen Bosque Muy Húmedo Tropical, en terrenos altos; Bosque Húmedo Tropical, en los terrenos más bajos, y Bosque Húmedo Tropical de Transición a Seco, al pie de los montes de la Fila Costeña. La temperatura promedio es de 22 a $23^{\circ} \mathrm{C}$, con temperaturas máximas promedio de $28^{\circ} \mathrm{C}$ y mínimas de $18^{\circ} \mathrm{C}$; la humedad relativa se estima en un 86 a $88 \%$ para los meses entre setiembre y diciembre.

La precipitación promedio anual varía entre 1700 y $1800 \mathrm{~mm}$. La estación lluviosa va desde abril a noviembre y las seca desde diciembre a marzo. En julio se presenta una disminución en la precipitación (canícula), durante la cual los productores realizan la cosecha del frijol.

\section{Caracterización de los principales agroecosistemas utilizados}

Uso de la tierra y diversificación: a pesar de que los suelos tienen aptitud para cultivos perennes o forestales, la mayoría de los productores cultivan granos básicos, los cuales diversifican en distintos grados (principalmente pastos, tiquisque y café) (Figura 2).

En las fincas pequeñas (hasta 5 ha) la mayoría de los productores producen sólo granos básicos, o diversifican a lo sumo con una actividad (tiquisque principalmente, café o pastos). Conforme aumenta el tamaño de la finca, el número de productores que sólo tienen granos básicos disminuye, y empiezan a aparecer más productores que diversifican; primero con tiquisque, luego con café y por último con pastos. A partir

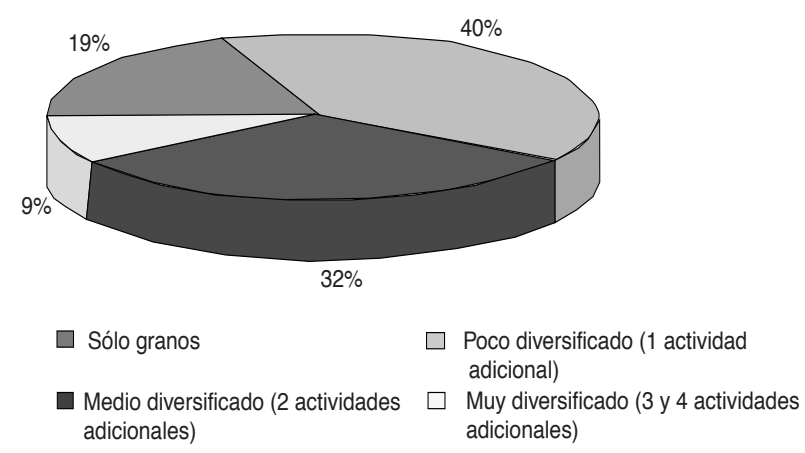

Figura 2. Diversificación de cultivos en la zona de Pejibaye, Pérez Zeledón, Costa Rica

de las fincas de 10 ha la diversificación prioriza el pasto y luego tiquisque, es decir con la mayor disponibilidad de área los productores prefieren la actividad ganadera antes que la agrícola para la generación de ingresos, esto se debe a que la actividad pecuaria requiere de mayor de tierra y es mucho menos riesgosa en términos climáticos. Esta tendencia se mantiene aún cuando las fincas son de más de 50 ha, y a lo sumo, se diversifica con cuatro actividades, es decir los productores concentran su quehacer en la ganadería, tiquisque y café, y prácticamente no se encuentran quienes se dedican sólo a granos básicos.

La producción de granos: situación y tendencias: La mayoría de los productores siembra menos de 5 ha de granos básicos (71\%), un $23 \%$ entre cinco y menos de 10 ha, mientras el restante $6 \%$ dedica entre más de 10 y hasta 50 ha.

Sólo una minoría de agricultores siembra exclusivamente un tipo de grano, puesto que un $85 \%$ diversifica el subsistema de granos básicos, siendo la combinación maíz/frijol y maíz/frijol/arroz mayoritaria entre los agricultores (Figura 3).

Maíz: El 90\% de los productores cultivan maíz durante la época veranera (veranera: de diciembre a febrero; inverniz: mayo a agosto), con un promedio de 2,5 ha y con rangos que van desde menos de un cuarto de hectárea y hasta 12 ha. El rendimiento promedio es de $1932 \mathrm{~kg} / \mathrm{ha}$ (rango de 23 a $5750 \mathrm{~kg} / \mathrm{ha}$ ). Cerca del 44\% de los productores han disminuido su área de maíz durante la época inverniz, así como han percibido una reducción en los rendimientos.

En el caso de la siembra de inverniz, la cantidad de agricultores que la practican se reduce a un $32 \%$, con una extensión promedio similar a la veranera $(2,1 \mathrm{ha})$. El rendimiento promedio es de $1978 \mathrm{~kg} / \mathrm{ha}$. En general los rendimientos en inverniz podrían ser mayores que 


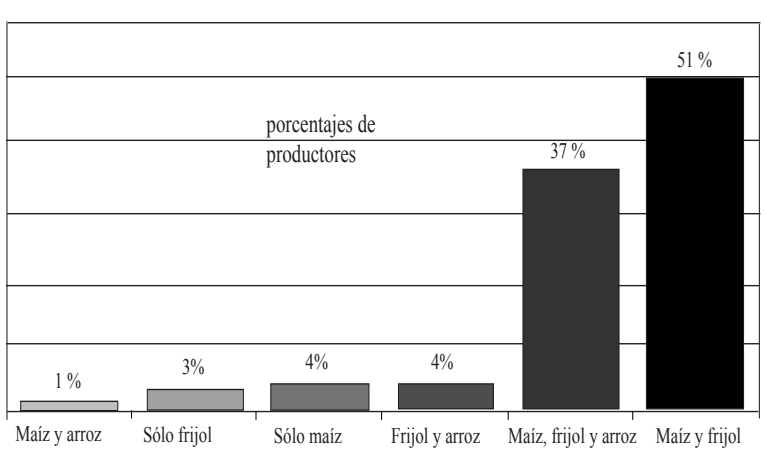

Figura 3. Combinación de cultivos de granos básicos en la zona de Pejibaye, Pérez Zeledón, Costa Rica.

en veranera, de no darse ataques de aves (pericos). Más de la mitad de los agricultores señalan que han disminuido las áreas destinados a maíz durante esta siembra, así como una caída en los rendimientos.

Las tendencias de la producción de granos señalan una reducción de áreas y de rendimientos, debido principalmente a las inestabilidades climáticas de los últimos años (ver el punto 4). En este sentido un 51\% de los productores redujeron sus áreas de siembra y un $63 \%$ vieron disminuidos los rendimientos.

Frijol: El 96\% de los productores tienen áreas promedio de 2,7 ha, con rendimientos promedio de 506 $\mathrm{kg} / \mathrm{ha}$.

Aunque es cultivado tanto en la época veranera como en la inverniz, la principal época es esta última, con un promedio de 3 ha y con rangos que van desde un cuarto de hectárea y hasta 25 ha; el $70 \%$ de los productores siembran hasta 3 ha. El rendimiento promedio es de $644 \mathrm{~kg} / \mathrm{ha}$. Casi la mitad (47\%) afirma haber reducido las áreas destinadas al frijol durante esa época de siembra.

En el caso de la siembra veranera, la cantidad de agricultores que la practican se reduce a un $47 \%$, con una extensión promedio inferior a la inverniz (2 ha). El rendimiento promedio es de $460 \mathrm{~kg} / \mathrm{ha}$.

Con las áreas y rendimientos las tendencias son similares al maíz, un $57 \%$ de productores disminuyeron las áreas de siembra y un $74 \%$ señalaron mermas en los rendimientos.

Uso de insumos: La mayoría de los productores utilizan insumos externos para obtener la producción de maíz (Cuadro 2). El uso generalizado de insumos se refleja tanto en los mayores rendimientos promedio alcanzados como en mayores costos monetarios por hectárea. Se observa una tendencia al incremento en los insumos externos con relación a hace cinco años.
Cuadro 2. Utilización de insumos externos en maíz.

\begin{tabular}{lc}
\hline Tipo de insumos & \% de productores \\
\hline Uso de fertilizantes & 96 \\
Uso de herbicidas & 96 \\
Uso de otros plaguicidas & 83 \\
Uso semilla mejorada & 69 \\
\hline
\end{tabular}

Analizando la situación del frijol (Cuadro 3), casi la totalidad de los productores utilizan insumos externos que se refleja tanto en los rendimientos promedio alcanzados como en mayores costos monetarios por hectárea. Al igual que en caso del maíz, se muestra una tendencia al aumento en el uso de insumos externos.

Cuadro 3. Utilización de insumos externos en frijol

\begin{tabular}{lc}
\hline Tipo de insumos & \% de productores \\
\hline Uso de fertilizantes & 96 \\
Uso de herbicidas & 98 \\
Uso de otros plaguicidas & 83 \\
Uso semilla mejorada & 68 \\
\hline
\end{tabular}

Fuente: IDEAS, datos de la investigación.

Destino de la producción: Con el maíz más del 95\% de la producción obtenida se destina a la venta (Figura 4). Considerando ambas siembras, en un $84 \%$ de los casos se vendió exclusivamente al comerciante, en un 10\% a una asociación de productores, y un $6 \%$ de los casos se entregó tanto al comerciante como a la asociación. En la época inverniz la venta al comerciante es mayor que durante la veranera, pues en la primera casi no existe participación de las asociaciones de productores en la comercialización del grano, por los bajos volúmenes de producción. El $84 \%$ de los productores guardan maíz para autoconsumo en veranera $(230 \mathrm{~kg})$ y el $44 \%$ guardan para semilla (46 kg); en inverniz el $61 \%$ guardan para su consumo unos $184 \mathrm{~kg}$, mientras para semilla un 39\% deja una reserva (69 kg en promedio).

En el caso del frijol, comparando por épocas de siembra, el $78 \%$ del frijol obtenido en la cosecha veranera se destina a la venta, inferior al caso de inverniz que alcanza $91 \%$, lo que señala que la producción de esta última tiene un objetivo más claramente destinado al mercado (Figura 5).

En un $75 \%$ de los casos la cosecha comercializada fue entregada al intermediario, en un $22 \%$ a la asociación de productores, y solamente en un $3 \%$ tanto al intermediario como a la asociación, situación que es similar para ambas cosechas. Del total del producto comercializado, entre un 26 y $29 \%$ es canalizado por medio de asociaciones de productores, las cuales tienen presencia en la zona en ambas cosechas. 


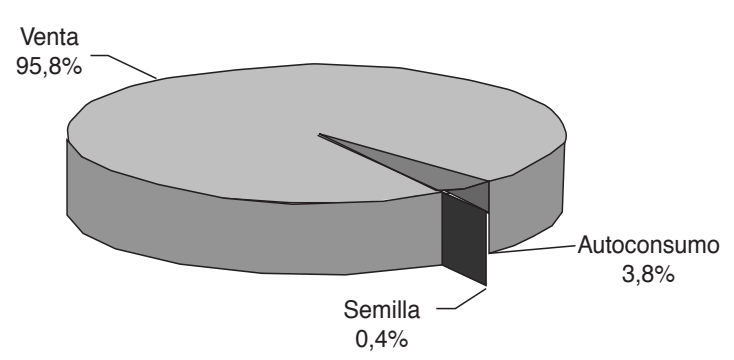

Epoca inverníz

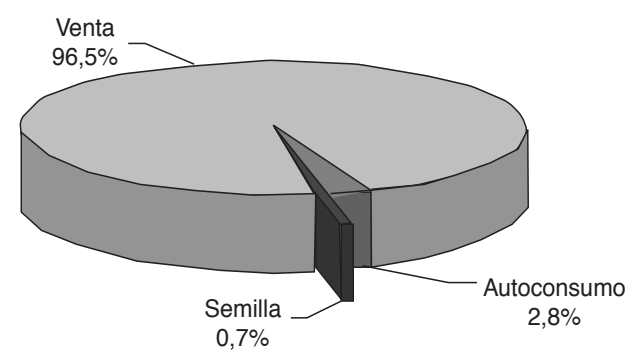

Epoca veranera

Figura 4. Destino de la producción de maíz a nivel de finca.

\section{Caracterización del rendimiento económico de prin- cipales prácticas productivas}

Costos de producción: Los datos por hectárea (Cuadro 4) indican costos similares para maíz y frijol; no obstante se perciben diferencias entre un mismo grano dependiendo de la época de siembra, donde las siembras consideradas más importantes tanto en términos de área como de destino del producto al mercado, presentan costos más altos para el maíz (veranero) y el frijol (inverniz). En el caso del frijol, los mayores costos en inverniz están relacionados con mayores rendimientos. En el maíz los costos por kg son similares, si bien resultan un poco superiores en la época de verano.

Destaca la importancia de la mano de obra familiar dentro de los costos de producción en ambos cultivos. Puede señalarse que ésta corresponde en promedio a un $56 \%$ del total de costos en el caso de maíz y a un 53\% en el frijol.

Rentabilidad de la producción: En general puede decirse que la producción de ambos granos resulta rentable en consideración de la relación beneficio/costo. Comparando ambos granos según los ingresos netos por hectárea, resulta más rentable la producción de frijol que la de maíz (Figura 6). Hay varias razones de sentido común que nos pueden ayudar a reforzar esta afirmación. Por ejemplo, se ven costos similares por hectárea para frijol inverniz y maíz veranero, donde el maíz tiene rendimientos tres veces superiores al frijol, pero a la vez el frijol tiene un precio por $\mathrm{kg}$ al menos cuatro veces superior al del maíz. Existen además otros costos no considerados en el estudio, que afectan la rentabilidad de los cultivos; por ejemplo el volumen de frijol que se obtiene es menor que el maíz, y por tanto se reducen los costos de secado y de transporte del producto.

\section{El impacto de las inestabilidades climáticas y la res- puesta de los productores}

En los últimos años las inestabilidades climáticas (como por ejemplo el fenómeno de "El Niño" o el Huracán Mitch) han dejado sentir sus efectos -en mayor o menor medida- casi en todas las actividades agropecuarias. En el caso de los granos básicos se han visto disminuidos los rendimientos y consecuentemente las áreas sembradas, lo cual ha hecho que los productores empiecen a desistir de ellos, y en algunos casos incursionen en otras actividades productivas.

Pese a la disminución de rendimientos y áreas en granos básicos, los productores continúan con estos

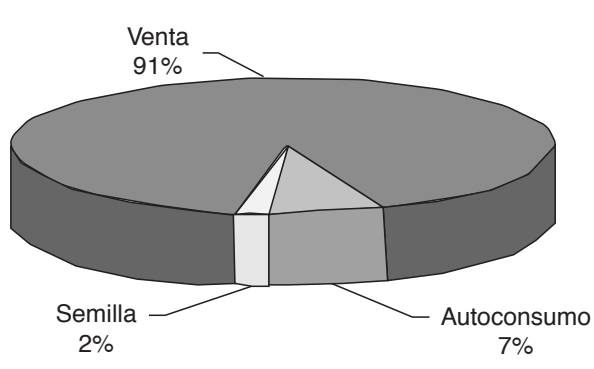

Epoca inverniz

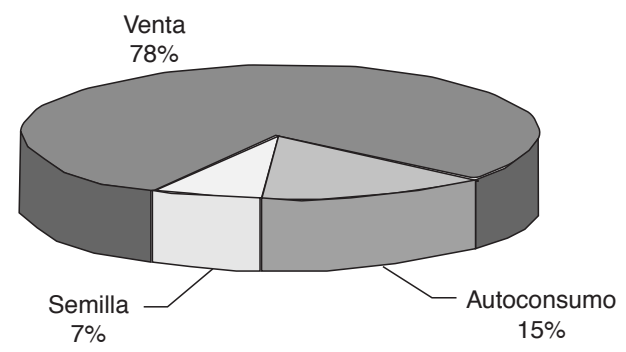

Epoca veranera

Figura 5. Destino de la producción de frijol a nivel de finca 
Cuadro 4. Costos de producción por hectárea

\begin{tabular}{cc}
\hline Cultivo & Costos $\mathbf{( \$ ) / h a ~}$ \\
\hline Maíz inverniz & 188 \\
Maíz veranero & 274 \\
Frijol inverniz & 247 \\
Frijol veranero & 183 \\
\hline
\end{tabular}

cultivos pues tienen una importancia económica que condiciona su permanencia dentro de los sistemas de producción. Para esto se vienen utilizando dos estrategias, relacionadas con cambios tecnológicos en los sistemas de producción: 1) la agricultura de Revolución Verde, y 2) la agricultura con un enfoque de manejo y uso sostenible de los recursos productivos.

En el primer caso, en los últimos años se vienen dando una serie de prácticas que tienden a incrementar los rendimientos en el corto plazo, y que están enmarcadas dentro de los paquetes tecnológicos tradicionalmente introducidos por la "Revolución Verde", concretamente el uso de maquinaría, uso de semilla mejorada, uso de fertilizantes, herbicidas y otros plaguicidas, los cuales son utilizados por la mayoría de los agricultores y que han tendido aumentar en los últimos cinco años.

Por otra parte se han empezado a utilizar otras técnicas alternativas a las convencionales, como cultivos de coberturas, abonos orgánicos, y diversas prácticas culturales, y agronómicas. Esto ha surgido como iniciativa de los mismos productores y en algunos casos promovidas por organismos de apoyo al sector agropecuario como el Ministerio de Agricultura y Ganadería (MAG) y el Programa Regional de Reforzamiento a la Investigación Agronómica sobre los Granos Básicos en Centroamérica (PRIAG) (PRIAG, 1996). Sin embargo su adopción no se ha generalizado, por ejemplo las prácticas tecnológicas para mantener y mejorar la fertilidad del suelo, asociadas a los granos básicos, no parecen lo suficientemente adoptadas como para detener la baja en los rendimientos, ante lo cual los agricultores tienden a incrementar el uso de paquetes tecnológicos intensivos en el uso de maquinaría, semillas mejoradas e insumos químicos, lo cual si bien puede funcionar en el corto plazo, no atacan el problema fundamental de pérdida de fertilidad del suelo, lo cual se incrementa dadas las condiciones de exceso de lluvia presentadas en la zona durante los últimos años. Por otro lado, la introducción del cultivo del tiquisque, el cual se siembra en terrenos limpios de cobertura debido a la mecanización, tiende a desmejorar la calidad de los suelos. Debido a esta última situación, se puede decir que lo avanzado en el cultivo de granos para mejorar las condiciones de manejo del suelo, podría revertirse si el cultivo del tiquisque se generaliza y se amplían sus áreas de siembra.
Otro elemento tecnológico empleado para paliar los efectos adversos del clima es la utilización de la diversidad de materiales genéticos, por lo general de origen local o criollo, pues éstas presentan particularidades en cuanto a adaptación a condiciones locales, hábitos de crecimiento favorables a condiciones topográficas, resistencia al clima, tolerancia a plagas y enfermedades o precocidad. En frijol destaca el uso de al menos dos variedades de grano rojo e igual número de grano negro, con el maíz, a pesar de que la diversidad genética no es tan amplia, todavía se emplean algunas variedades locales (de grano amarillo principalmente).

La conservación de semillas es una estrategia que ayuda a enfrentar situaciones de riesgo climático, consecuencia de lo cual los productores corren menos riesgo de comprometer su producción de granos en el caso de que se pierdan las cosechas, es decir pueden reiniciar la producción especialmente si se trata de variedades locales. En este punto cabe mencionar el importante papel que juegan dos asociaciones de productores (en Concepción de Pilas y Veracruz) en la producción de semilla de frijol, pues garantizan el abastecimiento local de las principales variedades utilizadas; esto mediante la compra de semilla a productores especializados en producirla, el almacenamiento, y la posterior distribución (venta) a los productores interesados.

\section{CONCLUSIONES}

En general se puede decir que la producción de ambos granos resulta rentable en consideración de la relación beneficio/costo. Comparando ambos granos bajo este indicador resulta más rentable la producción de frijol que la de maíz. Es así que el frijol es más importante que el maíz en términos del área.

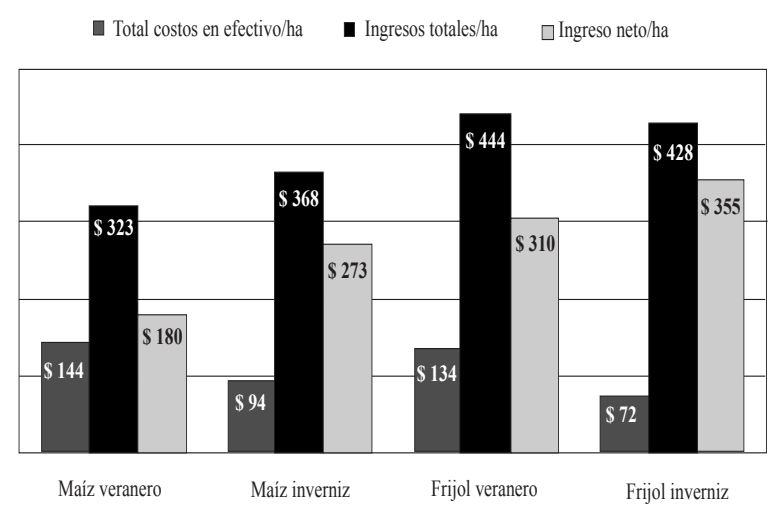

Figura 6. Rentabilidad de la producción de maíz y frijol [Los costos en efectivo por hectárea no consideran la mano de obra familiar (no remunerada)]. 
Destaca el hecho de que la mayoría de productores usan varios materiales de maíz y frijol. Sólo una minoría de agricultores siembra únicamente un tipo de grano. La combinación maíz/frijol y maíz/frijol/arroz es mayoritaria entre los agricultores.

Los agricultores de frijol destinan una parte superior de su cosecha para su propio consumo y semilla, y una proporción mayor se vende a las asociaciones de productores. Esta situación deriva en el uso de distintas variedades, unas para comercialización y otras para consumo.

Los entornos económicos (bajos precios) y climáticos (huracanes) han hecho que se de una tendencia a la reducción de los cultivos de maíz y frijol básicos en términos de área y rendimiento. De alguna u otra manera los productores de granos básicos han respondido a cambios en el entorno, en algunos casos con más éxito (uso de variedades) que en otros (comercialización). En esto se ve una integración de estrategias para enfrentar entorno económico y climático. Concretamente el uso de variedades rojas y negras en frijol, y prácticas tecnológicas alternativas a la convencionales, por ej. coberturas, abonos verdes. De manera complementaria, el fitomejoramiento participativo se convierte en una importante estrategia para el fortalecimiento de la diversidad varietal que está siendo utilizada para enfrentar el entorno.

En el caso del maíz, el mercado con un sólo comprador impacta sobre el uso de variedades. Se está dan- do que una empresa influye en el tipo de maíz que se siembra, condicionado a las características que demanda para ser procesado. En frijol sucede algo similar, el mercado demanda homogeneidad del producto, en términos de color y tamaño del grano. Es decir para ambos cultivos, las exigencias del mercado y empresas compradoras tienen un efecto sobre la biodiversidad. Pero también el libre mercado ha permitido que la diversidad genética de frijol adquiera valor económico diferenciado, especialmente para las variedades de color rojo las cuales se pagan mejor, esto viene a reforzar las variedades locales pues tradicionalmente, las mejoradas habían sido de color negro.

\section{LITERATURA CITADA}

ALVAREZ, P.; MORA, M. 1994. Sistemas locales de semillas: Maíz y Frijol. IDEAS, San José, Costa Rica. p.67

GAMBOA, M.; QUIROS S. 1998. Lineamientos para una Estrategia de desarrollo agropecuario. MAG. s.n.t.

MURILLO, M.; MORA, C. 1996. Situación y perspectivas de los granos básicos en Costa Rica. s.n.t

PROGRAMA DE REFORZAMIENTO A LA INVESTIGACIÓN AGRONÓMICA SOBRE LOS GRANOS BÁSICOS EN CENTROAMÉRICA (PRIAG). 1996. Encuesta informal agrosocieconómica de la Región Brunca, Costa Rica: Informe Preliminar. San José, C.R. s.n.t. 